\title{
Neue orale Antikoagulanzien
}

\section{Bei lebensbedrohlichen Blutungen ein Problem}

\begin{abstract}
Neue orale Antikoagulanzien haben die Antikoagulation einfacher und sicherer gemacht. Bedeutende Nachteile der neuen Substanzen sind aber, dass ein Monitoring und eine Antagonisierung nicht möglich sind. Dies kann vor allem bei schweren lebensbedrohlichen Blutungen zu Problemen führen.
\end{abstract}

Die Einführung der neuen oralen Antikoagulanzien, also des Thrombininhibitors Dabigatran und des Faktor-Xa-Inhibitors Rivaroxaban, hat zur Folge, dass immer mehr Patienten, vor allem jene mit Vorhofflimmern, eine hochwirksame Antikoagulation erhalten. „Doch der entscheidende Nachteil ist, dass ein Monitoring und eine Antagonisierung nicht möglich sind", sagt PD Jürgen Koscielny, Institut für Transfusionsmedizin, Berliner Charité. Bisher gäbe es keine Leitlinien zur Reversierung, weshalb das perioperative Blutungsrisiko ansteigen könne. Schwerwiegende Blutungskomplikationen traten mit den neuen oralen Antikoagulanzien bei etwa 3\% der Patienten auf - deutlich weniger als mit Vitamin-K-Antagonisten, die mit einem Blutungsrisiko von $8 \%$ assoziiert sind. Pharmakologischen Daten zufolge hat sich das Gerinnungssystem nach doppelter Halbwertzeit (Rivaroxaban/Apixaban: 20-30 Std.; Dabigatran 24-48 Std.) weitgehend spontan erholt. Dann nämlich liegt die pharmakodynamische Aktivität $<25 \%$. „Bei Patienten mit höhergradig eingeschränkter Nierenfunktion muss man mit Dabigatran aber 48-96 Stunden einplanen", so Koscielny. Doch bei einer schweren akuten lebensbedrohlichen Blutung könne man auf eine Spontanerholung nicht warten.

Eine schwere vital bedrohliche Blutung liegt vor, wenn ein persistierender Transfusionsbedarf über 6 Erythrozytenkonzentrationen in 12 Stunden besteht und/oder bei einer hämodynamischen Instabilität oder Katecholaminpflicht. Dabei kann es sich um eine intrazerebrale/intraspinale Blutung, um eine schwere Blutung in präformierte Körperhöhlen, eine Organblutung mit drohendem Organausfall, um eine schwere Kompartiment-Blutung, besonders in den Extremitäten, oder eine Blutung in die Halsweichteile mit Erstickungsgefahr handeln.

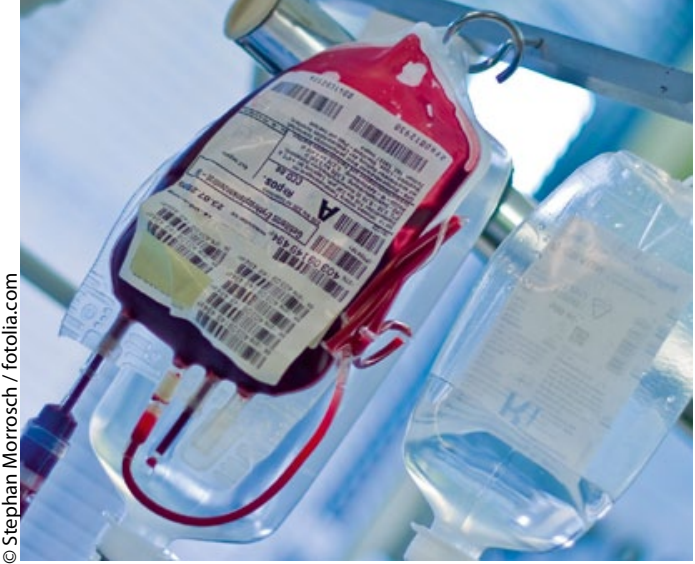

Schwere Blutungen können mit den neuen oralen Antikoagulanzien problematisch sein. Lebensrettend könnte dann unter anderem eine Blutransfusion wirken.

\section{Kein zuverlässiges Labormonitoring} Gerade in solchen heiklen Situationen wären bei den neuen oralen Antikoagulanzien zuverlässige Gerinnungstests vonnöten, um ein spezielles Hämostase-Management zu ermöglichen. „Das größte Problem ist, dass sie zwar die Globaltests für die Gerinnung verändern, aber keine Information über die Intensität der Antikoagulation und damit über deren Wirksamkeit oder ihr Blutungsrisiko liefern", so Koscielny. Der Thrombinhemmer verändert vorrangig die aktivierte partielle Thromboplastinzeit (aPTT) und Thrombinzeit (TZ). Die Faktor-Xa-Inhibitoren dagegen verlängern vor allem die Prothrombinzeit (PT) und damit den Quickwert und INR. Beides ist abhängig von der momentanen Plasmakonzentration des Wirkstoffs, sprich 2-3 Stunden nach Einnahme sind sie am höchsten. „Bei schweren Blutungen sollte deshalb primär immer gefragt werden, wann die Substanz zuletzt eingenommen wurde", rät Koscielny. Der Zusammenhang zwischen Plasmakonzentration und Gerinnungswerte hänge zudem stark vom Testsystem ab und sei in der klinischen Routine ohne Aussage.

Auch sind wegen der großen therapeutischen Breite die Plasmaspiegel und die Wirksamkeit bzw. Gefährlichkeit derTherapie nicht assoziiert.,"Auch wenn eine lineare Korrelation zwischen der Anti-Xa-Aktivität und den Rivaroxaban-Plasmaspiegeln besteht, darf man nicht davon ausgehen, dass die antithrombotische Wirkung mit der antikoagulatorischen einhergeht", so Koscielny. Wegen dieser Probleme sollte man möglichst wenig Parameter bestimmen, bei Dabigatran nur die TP und TZ, bei Rivaroxaban und Apixaban die TP, aPTT und Anti-Xa-Aktivität.

\section{Hämostase-Management}

Hat ein Patient mit einem neuen oralen Antikoagulanz eine leichte Blutung, sollte lediglich die nächste Einnahme verzögert oder das Mittel kurzfristig abgesetzt werden. Bei mittelschweren bis schweren Blutungen sollte primär die mechanische Kompression bzw. chirurgische Intervention zur Blutstillung ausgeschöpft werden. Wichtig ist, eine ausreichende Diurese sicherzustellen und, falls nötig, die Gabe von Bluttransfusionen bzw. gefrorenem Frischplasma. Bei Dabigatran ist die Elimination mit einer Hämodialyse möglich, bei den kleinen molekularen Faktor-Xa-Inhibitoren jedoch nicht.

Bei vital bedrohlichen Blutungen ist ein spezielles Hämostase-Management mit Gabe von Plasmaderivaten erforderlich, die im Sinne eines therapeutischen Stufenkonzepts eingesetzt werden sollten. Initial empfiehlt es sich, PPSB in einer Dosis von 20-40 IE kg Körpergewicht (KG) zu geben. Kann die Blutung nach 0-15 Minuten nicht gestillt werden, sollte ein zweiter Bolus verabreicht werden. Ist das ohne Erfolg, kommt bei Rivaroxaban oder Apixaban rFVIla (90-120 $\mu \mathrm{g} /$ kg KG) zum Einsatz, bei Dabigatran aPPSB in einer Dosis von 50 bis maximal 200 IE kg KG/ Tag. Wirkt das auch nicht, kommt als ultima ratio bei Apixaban oder Rivaroxaban aPPSB und bei Dabigatran rFVIla zum Einsatz. „Angesichts fehlender Studiendaten basieren die Empfehlungen jedoch vorrangig auf Fallberichten", so Koscielny. Die Reversierung der Antikoagulation mit den angegebenen Plasmaderivaten sei deshalb "off label".

Dr. med. Peter Stiefelhagen, Hachenburg II

II Quelle: 11. Expertentreffen: Niedermolekulare Heparine - up to date

2012,13.10.2012 in Berlin 